\title{
СКРИНИНГ УРОВНЯ ИРФ-1 У ПАЦИЕНТОВ С АДЕНОМАМИ ГИПОФИЗА И ГИПЕРПРОЛАКТИНЕМИЕЙ
}

\author{
Уханова Ю.А., Иловайская И.А.
}

ГБУз МО «Московский научно-исследовательский клинический институт им. М.Ф. Владимирского», Москва

ЦЕЛЬ: определить необходимость скрининга уровня ИРФ-1 у пациентов с аденомой гипофиза без явных симптомов акромегалии, сопровождающейся синдромом гиперпролактинемии.

МАТЕРИАЛЫ И МЕТОДЫ: бЫЛо Проведено пилотное одномоментное исследование на основании анализа медицинской документации пациентов с подтвержденной аденомой гипофиза в сочетании с гиперпролактинемией, которые обратились в КДЦ МОНИКИ им. М.Ф. Владимирского. Всего в исследование вошло 114 пациентов: мужчин - 33 (28,9\%), женщин - 81 (71,1\%), с макроаденомами 61 (53,5\%), с микроаденомами - 53 (46,5\%). Медиана уровня пролактина составила у мужчин 2469 мME/мл $(519,14 ; 104700)$, у женщин - 2530 мME/мл $(596 ; 95106,3)$. У данной группы пациентов проводилась оценка частоты исследования уровня ИРФ-1, частоты выполнения пробы с нагрузкой глюкозой с определением уровня СТГ в 5 точках пациентам с повышенным уровнем ИРФ-1, а также частоты выявления случаев акромегалии.

РЕЗУЛЬТАТЫ: всего уровень ИРФ-1 был определен у 51/114 пациентов (44,7\%), из ниху 41/51 пациентов $(80,4 \%)$ - в дебюте заболевания, у 10/51 пациентов (19,6\%) - в связи с отсутствием нормализации уровня пролактина на фоне лечения максимальными дозами каберголина. Среди пациентов с известным уровнем ИРФ-1 его повышение зафиксировано у 10/51 (19,6\%) - (включая 7/41 и 3/10 соответственно). Проба с нагрузкой глюкозой была проведена всем 10 пациентам, по результатам которой диагноз акромегалии был подтвержден у 4 пациентов: 3 - в дебюте заболевания (2 женщины с микроаденомой, 1 женщина с макроаденомой), 1 - на фоне лечения каберголином (1 женщина с макроаденомой).

ВывОды: в реальной клинической практике уровень ИРФ-1 был определен менее чем у половины $(44,7$ \%) пациентов с опухолями гипофиза и гиперпролактинемией. При этом доля выявленных пациентов с акромегалией среди обследованных больных с опухолями гипофиза и гиперпролактинемией, но без явных клинических признаков акромегалии, составила 7,8\% (4/51), что является высоким показателем для селективного скрининга акромегалии. Скрининг уровня ИРФ-1 может быть оправдан у пациентов с аденомами гипофиза и гиперпролактинемией.

КЛЮЧЕВЫЕ СЛОВА: ИРФ-1; акромегалия; гиперпролактинемия; аденома гипофиза. 